\title{
Duhamel versus transanal endorectal pull through (TERPT) for the surgical treatment of Hirschsprung's disease
}

\author{
E. Arts ${ }^{1} \cdot$ S. M. B. I. Botden ${ }^{1}$ M. Lacher ${ }^{2}$ P. Sloots ${ }^{3}$ - M. P. Stanton ${ }^{4}$.

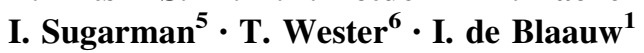

Received: 6 April 2016/Accepted: 6 April 2016/Published online: 14 September 2016

(c) The Author(s) 2016. This article is published with open access at Springerlink.com

\begin{abstract}
For the surgical treatment of Hirschsprung's disease, several surgical techniques are used to resect the distal aganglionic colon. Two frequently used techniques are the Duhamel procedure and the transanal endorectal pull-through procedure. During the '8th Pediatric Colorectal Course' in Nijmegen, November 2015, a workshop was organized to share experiences of both techniques by several experts in the field and to discuss (long term) outcomes. Specifically, the objective of the meeting was to discuss the main controversies in relation to the technical execution of both procedures in order to make an initial assessment of the limitations of available evidence for clinical decision-making and to formulate a set of preliminary recommendations for current clinical care and future research.
\end{abstract}

I. de Blaauw

ivo.deblaauw@radboudumc.nl

1 Department of Surgery, Division of Pediatric Surgery, Radboudumc-Amalia Children's Hospital, Nijmegen, The Netherlands

2 Department of Pediatric Surgery, University of Leipzig, Leipzig, Germany

3 Department of Pediatric Surgery, ErasmusMC-Sophia Children's Hospital, Rotterdam, The Netherlands

4 Department of Pediatric Surgery, University Hospital Southampton General Hospital, Southampton, UK

5 Department of Pediatric Surgery, Leeds Teaching Hospitals NHS Trust, Leeds, UK

6 Department of Pediatric Surgery, Karolinska University Hospital, Solna, Stockholm, Sweden
Keywords Hirschsprung's disease/surgery · Infant · Newborn · Proctocolectomy · Restorative/methods . Management of Hirschsprung's disease

\section{Introduction}

Hirschsprung's disease (HD) is a congenital condition that is caused by the absence of ganglion cells in the submucosal and myenteric plexuses of the distal intestine [1,2]. The surgical management of HD has moved from multistage open procedures to single-stage transanal surgical techniques [3]. Two frequently used techniques are the Duhamel retrorectal pull-through procedure $[4,5]$ and the transanal endorectal pull-through (TERPT) procedure [6]. Both techniques can involve laparoscopy as a means for taking biopsies to identify the transition zone and for mobilizing the colon [5]. In the Duhamel technique, a section of aganglionic rectum is left connected to a segment of ganglionic colon (side-to-side) as a pouch reservoir, whereas in the TERPT technique a very low direct anastomosis is made just above the dentate line $[6,7]$. The latter can be done by leaving a muscular rectal cuff (Soavelike) or with a full-thickness resection of the distal colon and rectum (Swenson-like). More than two decades have passed since the implementation of the laparoscopic Duhamel and TERPT techniques as the treatment strategies for HD. However, there is an ongoing debate about many of the key issues, such as which technique is preferable and the execution and timing of these procedures. It is unclear if one of these techniques yields significantly better general and disease-specific outcomes.

The TERPT procedure, first developed by De La Torre and Langer, was a Soave-like transanal submucosal dissection with an endorectal pull-through, leaving an 
aganglionic rectal muscular cuff $[6,7]$. This surgical procedure has also been modified to a transanal Swenson-like operation which does not require dissection in the submucosal plain but a straight resection of the full-thickness colon just above the dentate line [8]. Furthermore, a TERPT can be precluded by an open, transumbilical or laparoscopic biopsy for localization of the transition zone. Laparoscopy is then often used for mobilization of the aganglionic distal sigmoid colon.

When the Duhamel procedure is performed, the distal part of the aganglionic colon (rectum) remains in situ. After the resection, the ganglionic colon is placed in the avascular retrorectal plane and stapled or sutured side-toside to the native aganglionic rectum $[9,10]$. This was initially an open procedure; however, since the 1990s it has been performed laparoscopically with good results [9]. It can be a fully laparoscopic procedure, or a Pfannenstiel incision can be used when making the anastomosis $[9,10]$.

In recent years some centres of paediatric surgery have transitioned from performing the Duhamel procedure to performing the TERPT procedure in almost all cases $[12,13]$, but most surgeons appear to stick to their preferred surgical technique. There is little evidence supporting the superiority of procedure either in general or in specific cases [7, 8, 11-17], although some authors prefer the Duhamel procedure in long-segment disease. The available studies in which the short- and long-term outcomes of both techniques are investigated and compared often involve small heterogeneous patient samples and are frequently based on a retrospective or observational design, a combination which is likely to lead to biased results.

During the 8th Pediatric Colorectal Course and Workshops, in Nijmegen, the Netherlands, in November 2015, a 'Surgical techniques \& outcomes in Hirschsprung disease' workshop was organized that focused on this topic. The aim of the meeting was to: (1) discuss the main controversies related to the technical execution of the TERPT and Duhamel procedures and (2) to formulate a set of preliminary recommendations for current clinical care and future research.

\section{Discussion}

Experiences with both techniques differed greatly between the expert panel and participants in the workshop. It was agreed, however, by both the experts and the participants that the currently available evidence, particularly with regard to long-term outcomes, appears to be insufficient to significantly impact clinical decision. The consultants' personal experience with a certain technique seems to have the strongest influence on the choice of treatment on each centre. In addition, there was a general consensus amongst experts that only highly skilled experienced surgeons could successfully perform the procedures, particularly the Duhamel procedure. It was agreed that a minimum of 20 cases was required to reach the plateau of the learning curve for each of the procedures, and generally training was not begun until fellow status was reached. Positive experiences with the Duhamel procedure that were shared by all experts were the limited anal stretching necessary for this procedure and the good visibility during the whole process. Also, the minimally invasive nature of the TERPT procedure and the good cosmesis were points of agreement.

In addition to these points of consensus, several controversial aspects of the surgical technique were discussed in the workshop; (1) the modus operandi for identifying the transitional zone by means of surgical biopsy, (2) the type of muscular cuff that is created in the TERPT technique and (3) the (native) pouch and/or spur left after removal of the aganglionic bowel using the Duhamel technique.

\section{Colonic biopsy}

Before the actual process of removing the aganglionic bowel can be initiated, the transition zone between ganglionic bowel tissue and non-functioning aganglionic bowel needs to be determined. The gold standard for the diagnosis of HD is a biopsy [18, 19], for which several approaches are available; submucosal or seromuscular and transmural or full-thickness biopsies. Overall, (submucosal) rectal suction biopsy is initially performed for diagnostic purposes before the final surgery (TERPT or Duhamel). Submucosal biopsies are reported to be accurate, although reported sensitivity and specificity rates are 81-93\% and 97-98\%, respectively [20-22].

During surgery (open or laparoscopy), a seromuscular or a transmural full-thickness biopsy can be taken. A punch biopsy that samples seromuscular bowel tissue only is regarded as a simple, safe, fast and inexpensive method, although this type of biopsy may be more difficult for the pathologist to interpret [20, 23, 24]. In cases of partial circumferential aganglionosis, severe myenteric hypoganglionosis or hypertrophic submucosal nerves, a transmural full-thickness biopsy is more appropriate [25]. The risk of taking an individual section that is not through-and-through (full thickness) is that one may be sampling ganglionic tissue while underneath in the submucosa the bowel tissue is aganglionic or may display signs of neural dysplasia [26]. Leaving (partly) non-functional bowel (transitional zone pull-through) may lead to long-term complications such as constipation, obstructive symptoms with enterocolitis and widening of the distal colon [26].

The experts' view on the preferred modus operandi for acquiring the biopsy during a TERPT or Duhamel procedure was the first point discussed during the workshop. The 
seromuscular biopsy was preferred by some experts since in their experience it had the advantage of a lower risk of spillage of bowel contents in the abdominal cavity and minimized the risk of perforation postoperatively. Several textbooks and published papers also mention seromuscular biopsies for diagnostics during laparoscopy [27], and the main advantage being that this method is minimally invasive and there is no spillage of bowel contents. The opinion of the experts was that the seromuscular biopsy may give more sampling errors if the transition zone is irregular.

A full-thickness biopsy on the other hand allows the pathologist to review all the layers of the bowel. Another method that can be used (particularly in open Duhamel procedures) is that of a circumferential doughnut biopsy taken intraoperatively. This allows the pathologist to review a sample in all quadrants rather than just the antimesenteric border, when a single-point biopsy is taken. This will certainly give the least errors. The potential benefit of a full-thickness biopsy or doughnut is that it will significantly decrease the number of patients with a transitional zone pull-through. Many of these patients require redo surgery although the exact numbers are not available [26]. The potential downside is a longer procedure every time a more proximal doughnut biopsy is required.

The experts also disagreed about the timing of the biopsy. TERPT is mostly initiated with a perineal dissection, and a biopsy is generally taken when a change in circumference or calibre of the colon is observed. However, several experts favoured a preliminary biopsy by means of laparoscopy or through an umbilical incision, before initiating the perineal dissection. The advantage of taking a preliminary biopsy is that it provides more certainty about the location of the transitional zone, which may be particularly important as this location may differ from the radiological findings $[19,28]$. Consequently, the length of the aganglionic section of the colon may be underestimated, which could complicate a fully transanal approach [29]. In the light of this issue, another advantage of a (laparoscopically assisted) preliminary biopsy was discussed, namely that whilst waiting for the analysis of the frozen section by a pathologist (at least 30-40 min), the surgeon can proceed with the mobilization of the colon distal to the biopsy intra-abdominally. This may reduce the stress on the anal sphincters and also allows for a visual check for torsion of the bowel.

\section{Rectal muscular cuff versus no cuff}

The original TERPT procedure described by De La Torre [6] and in a separate study by Langer [8] was a Soave-like procedure requiring a muscular rectal cuff to be dissected from the submucosa transanally. It can be technically challenging but was originally implemented to protect the surrounding tissue from surgical damage. More recently, a modification of the TERPT technique was described which involves going straight, full thickness, not mobilizing a rectal cuff by dissecting the submucosal plane (Swensonlike technique) [30, 31]. Several surgical key remarks were made on the subject. First of all, very often the procedures are started as a Soave-like procedure with a submucosal surgical plane in the first $0.5-1.0 \mathrm{~cm}$ and is then converted to full-thickness plane (Swenson-like technique), which does not require further submucosal dissection. However, it was observed that when using the Swenson plane, there is a risk that the surgeon inadvertently creates a plane too far from the rectum, gets lost and risks damaging structures anterior to the rectum (pelvic nerves, vagina, urethra/ bladder neck).

Another point of discussion was the length of the cuff in the original De La Torre and Langer technique. The original descriptions of TERPT by Soave include a submucosal dissection above the peritoneal reflection to prevent injury to pelvic structures [6]. However, this approach, proposed by Soave, was not a transanal technique but was performed transabdominally. Pelvic floor structures were easily damaged if the surgeon did not operate flush on the rectal wall, and therefore, the idea of leaving the rectal outer wall as a cuff was born. In the transanal technique, the submucosal dissection starts just above the dentate line and originally ends at the peritoneal reflection [6, 7]. To prevent postoperative obstruction, it was reported by several experts that they would incise the rectal cuff, which was also described by Yang et al. [3, 32].

One of the major issues that were discussed by the participants was the length of the rectal cuff. A complete rectal cuff (from above the dentate line to the peritoneal reflection) may constrict the pull-through bowel because of its obvious aganglionic nature, even when it is incised at the end of the procedure. It has also been described in the literature that a long seromuscular cuff should be avoided as it can lead to obstruction, constipation, constriction and enterocolitis [33, 34] and this was the experts' opinion as well. Determining the exact size or the ideal length of the cuff is not easy, but it was proposed that it should be no longer than at least $5 \mathrm{~cm}$. This opinion is very subjective as in some studies the comparison of longer and shorter cuff lengths showed that the difference in associated postoperative complications was not statistically significant $[33,35]$. Overall, at this meeting, the majority preferred using the Swenson approach for the TERPT procedure with a short cuff.

\section{Dysfunctional pouch}

In the Duhamel procedure, a pouch is made with aganglionic tissue on the ventral side and ganglionic tissue on 
the dorsal side, whereas in the TERPT procedure almost all aganglionic tissues are removed and an end-to-end anastomosis is made $1 \mathrm{~cm}$ above the dentate line $[9,10]$. It was discussed that the Duhamel technique may lead to specific complications, because of the native aganglionic rectal segment that remains in the new pouch after the reattachment to the healthy colon. As reported during the workshop, reattachment is performed by closing the top end of the native rectum and attaching the ganglionic bowel to the latero-posterior side of the aganglionic bowel making a distal pouch. However, it may leave enough tissue for a spur to be formed, which could impair proper bowel function. The preferred method was reported as stapling the opening of the pouch top to the wall of the pulled-through bowel, but incising the anterior wall of the pulled-down bowel and anastomosing this to the top of the pouch prevent pouch formation. The anterior wall of this pouch contains aganglionic tissue, which, if a spur is present, allows stool to sit there and lead to the anterior pouch expanding over time. A dilated pouch or a pouch that is left too long may lead to stasis of faeces, bacterial overgrowth, infection, obstruction and soiling [26, 36, 37]. In the experience of the attending experts, most of these mild symptoms generally improve. However, in more severe cases a redo operation is necessary, in which the Duhamel pouch is reduced in size $[27,37,38]$ or has to be removed and converted to a TERPT [38]. Thus, the Duhamel pouch remains a reason for concern in a few cases for some of experts in the field.

\section{Long-term outcomes}

Four long-term complications were considered essential by experts in patients with HD: obstructive symptoms (due to stricture, residual aganglionosis or transition zone, residual fibrosis or cuff tissue, torsion of distal colon), enterocolitis, incontinence and soiling. The experiences with these complications differed greatly amongst experts. The skill level of the surgeon was deemed of great importance for the success of the procedure and outcomes during longterm follow-up. There is only limited data available on long-term functional results after Duhamel or TERPT procedures. Systematic reviews by several groups report on research that explores outcomes of the Duhamel and TERPT techniques and predominantly include small, observational studies with limited follow-up length and heterogeneous patient samples [13, 39, 40]. Limitations in the quality of study design make it difficult to interpret results [40]. Also, in the opinion of the experts, the definitions of outcome parameters such as surgical complications, enterocolitis, constipation and incontinence differ greatly in the literature are not standardized for (longitudinal) monitoring of the outcome. There was a general consensus that the majority of HD patients experience problems with bowel function during follow-up, regardless of the technique that is used. Most experts said they find it difficult to properly monitor bowel function objectively during long-term follow-up as the child ages due to lack of a suitable measurement tool. There are many different types of outcome measures available such as the Rintala, Holschneider and Kelly scoring tools [41] or the enterocolitis definition proposed by Pastor et al. [42]. However, not all of these measurement tools are properly validated in the HD population and there does not appear to be one single scoring system that is generally accepted [41]. There are many well-validated bowel function scoring systems for adults, which may be of some use in assessing outcomes of adolescents in terms of soiling, for example, although it would be essential for paediatric surgeons to agree which is most appropriate.

Bias in the reporting of outcomes may exist, since in the studies it is often operating surgeon who monitors longterm outcome [41]. Interestingly, it was discussed that despite the possible source of bias, this set-up also has advantages as it guarantees continuity of care and benefits the patient-doctor relationship. Most participants strongly preferred that set-up. On the other hand, there was a general consensus about the need for an objective, standardized HD-specific scoring system for the monitoring of long-term outcomes during follow-up that preferably also touches on quality of life related to general health or gastrointestinal symptoms [43]. This may require someone not directly involved in the treatment to assist with follow-up assessment and the development and validation of a HDspecific instrument for long-term monitoring [41].

\section{Conclusions}

Although the evolution of surgical techniques in the treatment of HD has provided tremendous improvement in patient outcomes, there are certain aspects of treatment that could be improved. As regards the Duhamel versus TERPT technique, evidence is insufficient to recommend one technique over the other and the surgeon's experience is the key factor determining the choice of procedure. Perhaps these techniques could be applied more efficiently in certain subgroups, which have to be determined. This expert workshop led to the recommendation for a systematic review on the technical execution of the Duhamel and TERPT procedures.

Summary of the main consensus points and recommendations:

- The experience of the main surgeon is likely to have a major effect on long-term outcomes. As there currently 
is no evidence to suggest one technique has significant superiority over another, the technique, the surgeon is most experienced with, is recommended.

- Duhamel or TERPT may be the best for specific subgroups of patients, but this needs further research and evidence. The TERPT technique may be preferable in straightforward cases, with a more distal localization of aganglionosis, and the Duhamel technique is favourable when treating patients with long-segment disease affecting the proximal colon.

- Laparoscopy is preferred over open techniques to assist in a Duhamel and/or transanal pull-through procedure.

- A preliminary biopsy (preferably full thickness) and laparoscopic intraabdominal mobilization of the proximal colon should be considered to reduce stress on the anal sphincter (TERPT) and permit proper localization of the transitional zone.

- There is a need for objective and clear definitions of both the surgical techniques and the primary long-term outcomes (bowel function) preferably including quality of life.

\section{Compliance with ethical standards}

Conflict of interest The authors declare that they have no conflict of interest.

Ethical approval This article does not contain any studies with human participants performed by any of the authors.

Informed consent For this type of study formal consent is not required.

Open Access This article is distributed under the terms of the Creative Commons Attribution 4.0 International License (http://crea tivecommons.org/licenses/by/4.0/), which permits unrestricted use, distribution, and reproduction in any medium, provided you give appropriate credit to the original author(s) and the source, provide a link to the Creative Commons license, and indicate if changes were made.

\section{References}

1. De Lorijn F, Boeckzstaens GE, Benninga MA (2007) Symptomatology, pathophysiology, diagnostic work-up, and treatment of Hirschsprung disease in infancy and childhood. Curr Gastroenterol Rep 9:245-253

2. Kaiser G, Bettex M (1982) Disorders and congenital malformations associated with Hirschsprung's disease. In: Holschneider AM (ed) Hirschsprung's disease. Verlag, Stuttgart, pp 49-53

3. De La Torre L, Langer JC (2010) Transanal endorectal pullthrough for Hirschsprung disease: technique controversies, pearls, pitfalls, and an organized approach to the management of postoperative obstructive symptoms. Semin Pediatr Surg 19:96-106

4. Stockmann PT, Philippart AI (1996) The Duhamel procedure for Hirschsprung's disease. Semin Pediatr Surg 31:901-902
5. Georgeson KE, Cohen RD, Hebra A et al (1999) Primary laparoscopic-assisted endorectal colon pull-through for Hirschsprung's disease: a new gold standard. Ann Surg 229:678-683

6. De La Torre-Mondragon L, Ortega-Salgado JA (1998) Transanal endorectal pull-through for Hirschsprung's disease. J Pediatr Surg 33:1283-1286

7. De La Torre L, Ortega A (2000) Transanal versus open endorectal pull-through for Hirschsprung's disease. J Pediatr Surg 35:1630-1632

8. Langer JC (2012) Laparoscopic and transanal pull-through for Hirschsprung disease. Semin Pediatr Surg 21:283-290

9. Travassos DV, Bax NM, Van der Zee DC (2007) Duhamel procedure: a comparative retrospective study between an open and a laparoscopic technique. Surg Endosc 21:2163-2165

10. Van der Zee DC, Bax NMA (1996) Duhamel-Martin procedure for Hirschsprung's disease in neonates and infants: one-stage operation. J Pediatr Surg 31:901-902

11. Giuliani S, Betalli P, Narciso A et al (2011) Outcome comparison among laparoscopic Duhamel, laparotomic Duhamel, and transanal endorectal pull-through: a single-center, 18-year experience. J Laparoendosc Adv Surg Tech A 21:859-863

12. Gunnarsdóttir A, Larsson LT, Arnbjörnsson E (2010) Transanal endorectal vs. Duhamel pull-through for Hirschsprung's disease. Eur J Pediatr Surg 20:242-246

13. Chen Y, Nah SA, Laskmi NK et al (2013) Transanal endorectal pull-through versus transabdominal approach for Hirschsprung's disease; a systematic review and meta-analysis. J Pediatr Surg 48:642-651

14. De Lagausie P, Berrebi D, Geib G, Sebag G, Aigrain Y (1999) Laparoscopic Duhamel procedure: management of 30 cases. Surg Endosc 13:972-974

15. Langer JC, Durrant AC, de la Torre-Mondragón L et al (2003) One-stage transanal Soave pullthrough for Hirschsprung disease: a multicenter experience with 141 children. Ann Surg 238:569-576

16. Ludman I, Spitz L, Tsuji H, Pierro A (2002) Hirschsprungs's disease: functional and psychological follow-up comparing total colonic and rectosigmoid aganglionosis. Arch Dis Child 86:348-351

17. Mills JL, Konkin DE, Milnder R, Penner JG, Langer M, Webber EM (2008) Long-term bowel function and quality of life in children with Hirschsprung's disease. J Pediatr Surg 43:899-905

18. Rahman N, Chouhan J, Gould S et al (2010) Rectal biopsy for Hirschsprung's disease. Are we performing too many? Eur J Pediatr Surg 20:95-97

19. Muller CO, Mignot C, Belarbi N, Berreibi D, Bonnard A (2012) Does the radiographic transition zone correlate with the level of aganglionosis on the specimen in Hirschsprung's disease? Pediatr Surg Int 28:597-601

20. Andrassy RJ, Isaacs H, Weitzman JJ (1981) Rectal suction biopsy for the diagnosis of Hirschsprung's disease. Ann Surg 193:419-424

21. De Lorijn F, Kremer LC, Reitsma JB, Benninga MA (2006) Diagnostic tests in Hirschsprung disease: a systematic review. J Pedatr Gastroenterol Nutr 42:496-505

22. Keyzer-Dekker CM, Sloots CE, Linschoten IK, Biermann K, Meeussen C, Doukas M (2016) Effectiveness of rectal suction biopsy in diagnosing Hirschsprung disease. Eur J Pediatr Surg 26:100-105

23. Alizai NK, Batcup G, Dixon MF, Stringer MD (1998) Rectal biopsy for Hirschsprung's disease: what is the optimum method? Pediatr Surg Int 13:121-124

24. Kapur RP, Kennedy AJ (2013) Histopathologic delineation of the transition zone in short-segment Hirschsprung disease. Pediatr Dev Pathol 16:252-266 
25. Szylberg L, Marszalek A (2014) Diagnosis of Hirschsprung's disease with particular emphasis on histopathology. A systemic review of current literature. Prz Gastroenterol 9:264-269

26. Lawal TA, Chatoorgoon K, Collins MH, Coe A, Peña A, Levitt MA (2011) Redo pull-through in Hirschsprung's disease for obstructive symptoms due to residual aganglionosis and transition zone bowel. J Pediatr Surg 46:342-347

27. Pena A, Elicevik M, Levitt MA (2007) Reoperations in Hirschsprung disease. J Pediatr Surg 41:1008-1014

28. Proctor ML, Traubici J, Langer JC et al (2003) Correlation between radiographic transition zone and level of aganglionosis in Hirschsprung's disease: implications for surgical approach. J Pediatr Surg 38:775-778

29. Marquez TT, Acton RD, Hess DJ, Duval S, Saltzman DA (2009) Comprehensive review of procedures for total colonic aganglionosis. J Pediatr Surg 44:257-265

30. Levitt MA, Hamrick MC, Eradi B, Bischoff A, Hall J, Peña A (2013) Transanal, full-thickness, Swenson-like approach for Hirschsprung disease. J Pediatr Surg 48:2289-2295

31. Li AW, Zhang WT, Li FH, Cui XH, Duan XS (2006) A new modification of transanal Soave pull-through procedure for Hirschsprung's disease. Chin Med J 119:37-42

32. Yang L, Tang S, Cao G et al (2012) Transanal endorectal pullthrough for Hirschsprung's disease using long cuff dissection and short V-shaped partially resected cuff anastomosis: early and late outcomes. J Pediatr Surg 28:515-521

33. Nasr A, Langer JC (2007) Evolution of the technique in the transanal pull-through for Hirschsprung's disease: effect on outcome. J Pediatr Surg 42:36-39

34. Dickie BH, Webb KM, Eradi B, Levitt MA (2014) The problematic Soave cuff in Hirschsprung disease: manifestations and treatment. J Pediatr Surg 49:77-80
35. Pratap A, Gupta DK, Shakya VC et al (2007) Analysis of problems, complications, avoidance and management with transanal pull-through for Hirschsprung disease. J Pediatr Surg 42:1869-1876

36. Bax MA (2006) Duhamel lecture: the incurability of Hirschsprung's disease. Eur J Pediatr Surg 16:380-384

37. Weber TR, Fortuna RS, Silen ML, Dillon PA (1999) Reoperation for Hirschsprung's disease. J Pediatr Surg 34:153-156

38. Chatoorgoon K, Pena A, Lawal TA, Levitt M (2011) The problematic Duhamel pouch in Hirschsprung's disease: manifestations and treatment. Eur J Pediatr Surg 21:366-369

39. Laughlin DM, Friedmacher F, Puri P (2012) Total colonic aganglionosis: a systematic review and meta-analysis of longterm clinical outcome. Pediatr Surg Int 28:773-779

40. Gosemann JH, Friedmacher F, Ure B (2013) Open versus transanal pull-through for Hirschsprung disease: a systematic review of long-term outcome. Eur J Pediatr Surg 23:94-102

41. Ure BM, Rintala RJ, Holschneider AM (2006) Scoring postoperative results. Anorectal malformations in children: embryology, diagnosis, surgical treatment, follow-up. Springer, Berlin, pp 351-359

42. Pastor AC, Osman F, Teitelbaum DH, Caty MG, Langer JC (2009) Development of a standardized definition for Hirschsprung's-associated enterocolitis: a delphi analysis. J Pediatr Surg 44:251-256

43. Varni JW, Bendo CB, Denham J et al (2015) PedsQL ${ }^{\mathrm{TM}}$ gastrointestinal symptoms scales and gastrointestinal worry scales in pediatric patients with functional and organic gastrointestinal diseases in comparison to healthy controls. Qual Life Res $24: 363-378$ 